\title{
Gaussian Thermal Operations and The Limits of Algorithmic Cooling
}

\author{
A. Serafini $\odot,{ }^{1}$ M. Lostaglio, ${ }^{2}$ S. Longden $\odot,{ }^{1}$ U. Shackerley-Bennett, ${ }^{1}$ C.-Y. Hsieh, ${ }^{2}$ and G. Adesso ${ }^{3}$ \\ ${ }^{1}$ Department of Physics \& Astronomy, University College London, Gower Street, WC1E 6BT, London, United Kingdom \\ ${ }^{2}$ ICFO_Institut de Ciències Fotòniques, The Barcelona Institute of Science and Technology, 08860 Castelldefels, Spain \\ ${ }^{3}$ School of Mathematical Sciences and Centre for the Mathematics and Theoretical Physics of Quantum Non-Equilibrium Systems, \\ University of Nottingham, University Park Campus, Nottingham NG7 2RD, United Kingdom
}

(Received 20 September 2019; revised manuscript received 29 November 2019; published 2 January 2020)

\begin{abstract}
The study of thermal operations allows one to investigate the ultimate possibilities of quantum states and of nanoscale thermal machines. Whilst fairly general, these results typically do not apply to continuous variable systems and do not take into account that, in many practically relevant settings, systemenvironment interactions are effectively bilinear. Here we tackle these issues by focusing on Gaussian quantum states and channels. We provide a complete characterization of the most general Gaussian thermal operation acting on an arbitrary number of bosonic modes, which turn out to be all embeddable in a Markovian dynamics, and derive necessary and sufficient conditions for state transformations under such operations in the single-mode case, encompassing states with nonzero coherence in the energy eigenbasis (i.e., squeezed states). Our analysis leads to a no-go result for the technologically relevant task of algorithmic cooling: We show that it is impossible to reduce the entropy of a system coupled to a Gaussian environment below its own or the environmental temperature, by means of a sequence of Gaussian thermal operations interspersed by arbitrary (even non-Gaussian) unitaries. These findings establish fundamental constraints on the usefulness of Gaussian resources for quantum thermodynamic processes.
\end{abstract}

DOI: 10.1103/PhysRevLett.124.010602

Introduction and summary.-The past few years have witnessed a resurgence of studies into the thermodynamics of quantum systems [1], which have lent novel insight into the nature of thermodynamic relations, as well as into the role of thermodynamic quantities such as temperature, entropy and work [2,3], set against the practical backdrop of realizing superior thermal machines operating in the quantum regime [4].

A key ingredient to any attempt to analyze these questions beyond the limited scope of a specific model is the characterization of a class of "thermal operations," i.e., of operations that can be realized with the aid of the surrounding environment [5,6] (for reviews, see $[7,8]$ ). Whilst the frameworks resulting from this approach may yield significant wisdom concerning the ultimate limitations that constrain thermal scenarios, they are at times fraught by a certain "lack of realism," in that they include interaction Hamiltonians which are not necessarily encountered in practice. Also, they are limited to finitedimensional settings. It is therefore desirable to single out and characterize subclasses of thermal operations with direct practical relevance.

To this aim, this Letter shall consider the subclass of Gaussian thermal operations (GTOs), i.e., the class of operations on continuous variable systems obtained by considering energy-preserving bilinear interaction Hamiltonians between the system and a thermal environment. This subclass is extremely relevant in practice, given that quadratic Hamiltonians, which generate Gaussian unitaries, are very common and that system-bath interactions are often linear or may be linearized, especially in quantum optics and analogous setups. Indeed, various experimental platform relevant to quantum thermodynamics operate in the Gaussian regime. Examples include cavity optomechanics $[9,10]$, Bose-Einstein condensates loaded into cavities [11], ions in harmonic traps $[12,13]$. In view of the same practical reasons, work extraction, storage and fluctuations [14-17], entropy production [18], heat transport [19], thermometry [20], and fluctuation-dissipation theorems [21] have been investigated in Gaussian scenarios. The capabilities of Gaussian operations in other, not necessarily thermodynamical, settings are also being considered [22,23].

The other defining feature of GTOs, alongside Gaussianity, is energy preservation. As we will prove, this feature implies that GTOs may be physically reproduced through operations corresponding, in the optical picture, to passive optical elements (i.e., semireflectant mirrors and dielectric plates), even in presence of thermal noise (which can be represented as a beam splitter coupling the input with a thermal mode). Alternately, dynamics equivalent to GTOs can be obtained by contact with a Markovian thermal reservoir (giving rise to the so-called quantum optical master equation, see $[24,25]$ ), or in coupled resonant cavities or optomechanical systems with negligible counter-rotating terms [26]. 
In this Letter, we shall achieve a compact, constructive characterization of the most general GTO on any number of modes. We shall see that such a characterization becomes particularly simple for systems with nondegenerate eigenfrequencies, where it can be cast as a single-mode property. We shall then derive necessary and sufficient conditions for state transformation on single-mode systems and then proceed to analyze the possibilities offered by algorithmic cooling in the Gaussian regime, through alternating GTOs and unitaries. We will prove that, at variance with the finitedimensional case [27], in the absence of ancillas no such strategy can cool the system below the environmental temperature. Sidebandlike strategies involving highfrequency ancillas or higher order interactions are necessary to such an aim. Also in view of the ubiquity of Gaussian evolutions as a complete toolbox for quantum technologies [28] and in the modeling of open quantum systems of harmonic systems, the fundamental limitations to cooling techniques we will establish in the Gaussian regime possess a direct practical interest.

Gaussian systems.-We will consider bosonic continuous variables encoded into vectors of self-adjoint operators $\hat{\mathbf{r}}=\left(\hat{x}_{1}, \hat{p}_{1}, \ldots, \hat{x}_{n}, \hat{p}_{n}\right)^{\top}$ obeying the canonical commutation relations $\left[\hat{\mathbf{r}}, \hat{\mathbf{r}}^{\top}\right]=i \Omega$, where the commutators are taken between all pairs of elements of $\hat{\mathbf{r}}$ (as in an outer product) and form the nondegenerate, antisymmetric symplectic form $\Omega$, with $\Omega=\Omega_{1}^{\oplus n}$ and $\Omega_{1}=\left(\begin{array}{cc}0 & 1 \\ -1 & 0\end{array}\right)$ [25]. A second-order Hamiltonian $\hat{H}$ is one that may be written as a second-order polynomial of $\hat{\mathbf{r}}: \hat{H}=\frac{1}{2}(\hat{\mathbf{r}}-\mathbf{d})^{\top} H(\hat{\mathbf{r}}-\mathbf{d})$ for a symmetric Hamiltonian matrix $H$ and a real vector $\mathbf{d}$. Gaussian states are then defined as the ground and (Gibbs) thermal states of second-order Hamiltonians, and are completely characterized by a vector of first moments $\mathbf{r}=$ $\langle\hat{\mathbf{r}}\rangle$ and the covariance matrix $(\mathrm{CM}) \boldsymbol{\sigma}=\left\langle\left\{(\hat{\mathbf{r}}-\mathbf{r}),(\hat{\mathbf{r}}-\mathbf{r})^{\top}\right\}\right\rangle$ where, again, the anticommutators $\{\cdot, \cdot\}$ are taken between each pair of operator entries to form the symmetric, real matrix $\sigma$, satisfying $\sigma+i \Omega \geq 0$ [25]. Gaussian unitary operations-ones that map Gaussian states into Gaussian states-are those generated by second-order Hamiltonians and admit a symplectic representation: their action on the second moments may be written as $\sigma \mapsto S \sigma S^{\top}$, where $S \in S p_{2 n, \mathbb{R}}$ (i.e., $S$ is such that $S \Omega S^{\top}=\Omega$ ). It is well known that any positive-definite real matrix $P$ may be put into "normal modes" by congruence with a symplectic transformation: $\exists S: S P S^{\top}=\bigoplus_{j=1}^{n} \nu_{j} \mathbb{1}_{2}$, where the $\nu_{j} \mathrm{~s}$ are the "symplectic eigenvalues" of $P$; if $P$ is a Hamiltonian matrix, the quantities $\nu_{j}$ represent the eigenfrequencies of $P$ (the frequencies of its normal modes). In the case of the $\mathrm{CM}$ of a quantum state, one has $\nu_{j} \geq 1$ (an expression of the uncertainty principle). Bear in mind that the spectrum of a Gaussian state is entirely determined by its symplectic eigenvalues and that tensor products at the Hilbert space level translate into direct sums in the Gaussian and phase space descriptions.
In the following, a major role will be played by the set of orthogonal symplectic transformations, for which $S S^{\top}=\mathbb{1}$ (also known as "compact" or "passive" transformations, as they do not require any source of energy in standard optical implementations). Further specific notation will prove convenient: we shall adopt the shorthand notation $S[\boldsymbol{\sigma}]=$ $S \sigma S^{\top}$ and the symbol $\operatorname{Tr}_{b}$ to denote partial tracing of the bath's degrees of freedom in the phase space, which just corresponds to pinching out the relevant part of a $\mathrm{CM}$, discarding the rest.

Let us also recall that the most general deterministic Gaussian CP map, obtained by letting the system interact with an environment in a Gaussian state through a quadratic interaction Hamiltonian, is characterized, up to arbitrary displacements of the first moments, by the mapping $\boldsymbol{\sigma} \mapsto X \boldsymbol{\sigma} X^{\top}+Y$, with $Y \geq-i X \Omega X^{\top}+i \Omega$ [25]. The first aim of this Letter will be characterizing the subclass of deterministic Gaussian CP maps that are also thermal. A particularly relevant class of single-mode channels, which will play a prominent role in what follows, is the so-called phase-covariant ones, where $X=x \mathbb{1}_{2}$ and $Y=y \mathbb{1}_{2}$, with $y \geq\left|1-x^{2}\right|[25,29]$, (throughout the Letter, the symbol $\mathbb{1}_{d}$ denotes the identity matrix in dimension $d$ ).

The class of Gaussian thermal operations.-Given a second-order system Hamiltonian $\hat{H}_{s}$ and an inverse temperature $\beta=1 /(k T)$ (where $k$ is the Boltzmann's constant and $T$ is the environment's temperature), we shall define GTOs as the operations obtained by (i) preparing an environmental ancilla with arbitrary second-order Hamiltonian $\hat{H}_{b}$ in the Gibbs state $e^{-\beta \hat{H}_{b}} / \operatorname{Tr}\left[e^{-\beta \hat{H}_{b}}\right]$, and (ii) letting the system and bath interact through an energy preserving Gaussian unitary $\hat{U}_{I}$ such that $\left[\hat{U}_{I}, \hat{H}_{s}+\hat{H}_{b}\right]=0$. The maps above arise naturally through contact with thermal reservoirs where the interactions are well described by polynomials of order two in the canonical operators, whose importance has been already remarked. Note that all energy preserving Gaussian unitaries can be written as $\hat{U}_{I}=e^{i \hat{H}_{I} t}$ for some $t \geq 0$ and $\hat{H}_{I}$ a Hamiltonian of order two in the canonical operators satisfying $\left[\hat{H}_{I}, \hat{H}_{s}+\hat{H}_{b}\right]=0$. Note also that the definition above coincides with the well-established definition of thermal operations $[5,6]$, once the restrictions to secondorder operations are lifted.

Arbitrary $\hat{H}_{s}, \hat{H}_{b}$, and $\hat{H}_{I}$ of order two are parametrized by the symmetric Hamiltonian matrices $H_{s}, H_{b}$, and $H_{I}$ and the vectors $\mathbf{d}_{s}, \mathbf{d}_{b}$, and $\mathbf{d}_{I}$. We will further restrict the Hamiltonian matrices of system and environment to be strictly positive. Hamiltonian matrices with negative eigenvalues correspond to Hamiltonian operators that are not bounded from below, and thus do not even admit a welldefined Gibbs state, so their exclusion is not a restriction. Positive semidefinite, but not strictly positive, Hamiltonian matrices correspond to a set of measure zero within the Gaussian realm, with Gibbs states that are not regular, traceclass Gaussian states and thus do not give rise to Gaussian 
CP-maps. It might still be possible to obtain legitimate operations from nonpositive system Hamiltonians, but we shall disregard such peculiar cases in this treatment.

First-order terms in the interaction Hamiltonian generate displacements (shifts in the first-moment vector $\mathbf{r}$ ). Since no first-order term commutes with a strictly positive quadratic Hamiltonian (linear displacements do affect the energy of trapped systems), displacements must be severely limited if they are to give rise to thermal operations. Rather than complicating our treatment with the inclusion of first-order terms, which do not add anything conceptually remarkable, we defer such a discussion to the Supplemental Material [30], and set all first-order terms $\mathbf{d}_{s}, \mathbf{d}_{b}$, and $\mathbf{d}_{I}$ to zero to present our main results.

Simulating Gaussian thermalizations. - Within the above restrictions, a GTO generally involves an arbitrary number of bath modes, as well as an arbitrary sequence of second order interactions between these and the system modes. A crucial question is then if there exists a simpler protocol able to reproduce every Gaussian thermalization with less extensive resources. Our first main result answers this question in the affirmative, presenting a very simple scheme able to simulate exactly a general GTO (recall the shorthand notation whereby symplectics act by congruence):

Theorem 1-Characterization of GTOs: Let $\hat{H}_{s}=$ $\frac{1}{2} \hat{\mathbf{r}}^{\top} H_{S} \hat{\mathbf{r}}$ be a system Hamiltonian with normal form $\bigoplus_{l} \omega_{l} \mathbb{1}_{2 n l}=S^{-1} H_{s} S^{\top-1}$, where $n_{l} \in \mathbb{N}$ is the mode degeneracy of the eigenfrequency $\omega_{l}$ and $S \in S p_{2 n, \mathbb{R}}$ for $n=\sum_{l} n_{l}$. The class of GTOs at background inverse temperature $\beta$ act on the system $\mathrm{CM} \sigma$ as

$$
\boldsymbol{\sigma} \mapsto S\left(\oplus_{l} W_{l} \circ \Phi_{l} \circ Z_{l}\left[S^{-1}[\boldsymbol{\sigma}]\right]\right),
$$

where the direct sum runs over the distinct eigenfrequencies and, setting $\nu_{l}=\left[\left(\mathrm{e}^{\beta \omega_{l}}+1\right) /\left(\mathrm{e}^{\beta \omega_{l}}-1\right)\right]$, (i) each $\Phi_{l}$ are phase-covariant CP maps [29], acting on the lth eigenfrequency space as $\Phi_{l}(\boldsymbol{\sigma})=X_{l} \boldsymbol{\sigma} X_{l}^{\top}+Y_{l}$, with $X_{l}=$ $\bigoplus_{k=1}^{n_{l}} \cos \theta_{l k} \mathbb{1}_{2}$ and $Y_{l}=\bigoplus_{k=1}^{n_{l}} \nu_{l} \sin ^{2} \theta_{l k} \mathbb{1}_{2}$, for $\theta_{l k} \in[0,2 \pi[$, and (ii) $W_{l}$ and $Z_{l}$ are passive symplectic transformations acting on the system's set of modes associated with the $l$ th eigenfrequency.

Let us now unravel this statement and the restrictions it poses on the structure of GTOs, which will also allow us to sketch the main lines of its proof (whose full details are found in the Supplemental Material [30]). The transformation $S$ is just the one bringing the system Hamiltonian into normal modes, set by the given system quadratic Hamiltonian [35].

The first step towards the statement above is realizing that, once both system and ancillas are cast into normal modes, all GTOs are obtained by letting the $n_{l}$ system modes pertaining to the same eigenfrequency $\omega_{l}$ interact with an equal number $n_{l}$ of environmental normal modes at the same frequency: $\left.\boldsymbol{\sigma} \mapsto S\left\{\operatorname{Tr}_{b}\left(O\left[S^{-1}[\boldsymbol{\sigma}] \oplus \boldsymbol{\sigma}_{b}\right)\right]\right)\right\}$, where $\sigma_{b}=\oplus_{l} \nu_{l} \mathbb{1}_{2 n l}$ and $O=\left(\oplus_{l} O_{l}\right)$, with each $O_{l}$ being a passive symplectic transformation acting on the system plus bath degenerate eigenfrequency subspace labelled by $l$ (of dimension $2 n_{l}$ ). Very significantly, normal modes belonging to different eigenfrequency sectors do not interact during thermal operations (this holds regardless of any correlations that may exist between the physical bath modes).

The second step to obtain the compact characterization above is that, due to the symmetries of the problem at hand, each $O_{l}$ admits a very simple structure:

$$
O_{l}=\left(W_{l} \oplus \mathbb{1}_{b}\right) \circ M_{l} \circ\left(Z_{l} \oplus \mathbb{1}_{b}\right),
$$

where, as already stated, $W_{l}$ and $Z_{l}$ are passive symplectic on the system, and $M_{l}$ is a set of beam splitters independently mixing each mode $j=1, \ldots, n_{l}$ with a corresponding mode of the environment: $M_{l}=R_{n_{l} n_{l}}^{(l)} \oplus \ldots \oplus R_{22}^{(l)} \oplus R_{11}^{(l)}$, where $R_{k k}^{(l)}$ denotes a beam splitter mixing system mode $k$ [with ladder operator $\hat{a}_{k}=\left(\hat{x}_{k}+i \hat{p}_{k}\right) / \sqrt{2}$ ] with bath mode $k$ (with ladder operator $\hat{b}_{k}$ ); at the Hilbert space level, $\hat{R}_{k k}^{(l)}=\mathrm{e}^{\left(\hat{a}_{k} \hat{b}_{k}^{\dagger}-\hat{a}_{k}^{\dagger} \hat{b}_{k}\right) \theta_{l k}}$.

Thus, in a GTO, each oscillator within the degenerate frequency sector is mixed with a correspondent thermal oscillator by means of a beam splitting operation. Tracing out the bath after such an interaction gives rise to the tensor product of phase-covariant channels that were denoted with $\Phi_{l}$. What is perhaps surprising is that every GTO can be simulated in this simple way, by independent interactions with the environmental modes. Besides, since the loss channels $\Phi_{l}$ are Markovian [25], the most general Gaussian thermalization can be generated by a simple Markovian master equation. Indeed, GTOs are the most common as well as easiest to implement transformations, corresponding, in the normal mode basis, to passive optics or loss to a thermal Markovian reservoir.

Single-mode criteria.-For each nondegenerate system eigenfrequency, a GTO reduces to a single-mode transformation. All single-mode passive transformations are phase shifters, and the transformation $Z_{l}$ may always be simplified by left multiplication with phase shifters (see [30]) and may thus, on a single mode, be reduced to the identity without loss of generality. Hence, the most general GTO on a nondegenerate eigenfrequency subspace takes a very simple form indeed.

Proposition 1-Single-mode GTOs: Let $\hat{H}_{s}=\frac{1}{2} \hat{\mathbf{r}}^{\top} H_{s} \hat{\mathbf{r}}=$ $(\omega / 2) \hat{\mathbf{r}}^{\top} S S^{\top} \hat{\mathbf{r}}$ be a single-mode system Hamiltonian, then the class of GTOs is given by

$$
\boldsymbol{\sigma} \mapsto S\left[p D_{\varphi} S^{-1} \boldsymbol{\sigma} S^{-1 \top} D_{\varphi}^{\top}+(1-p) \nu_{b} \mathbb{1}_{2}\right] S^{\top},
$$

with $p \in[0,1], \quad \nu_{b}=\left[\left(\mathrm{e}^{\beta \omega}+1\right) /\left(\mathrm{e}^{\beta \omega}-1\right)\right]$ and $D_{\varphi}=$ $\left(\begin{array}{c}\cos \varphi \sin \varphi \\ -\sin \varphi \cos \varphi\end{array}\right)$.

We can now spell out the full criterion for Gaussian state transformations through single-mode GTOs. That is, given 
an input $\mathrm{CM} \boldsymbol{\sigma}_{i}$ and an output $\mathrm{CM} \boldsymbol{\sigma}_{f}$, is there a GTO mapping $\boldsymbol{\sigma}_{i}$ into $\boldsymbol{\sigma}_{f}$ ? Here, one should recall that the most general single-mode $\mathrm{CM} \sigma$ may be written as a rotated and squeezed thermal state: $\boldsymbol{\sigma}=\nu D_{\varphi} \operatorname{diag}(z, 1 / z) D_{\varphi}^{\top}$, for $\varphi \in[0,2 \pi[, z \geq 1$ and $\nu \geq 1$.

Proposition 2-Single-mode state transformations: Let $\hat{H}_{s}=\frac{1}{2} \hat{\mathbf{r}}^{\top} H_{s} \hat{\mathbf{r}}=(\omega / 2) S S^{\top}$ be a single-mode system Hamiltonian. An initial Gaussian state with $\mathrm{CM} \sigma_{i}=$ $\nu_{i} S D_{\varphi_{i}} \operatorname{diag}\left(z_{i}, 1 / z_{i}\right) D_{\varphi_{i}}^{\top} S^{\top}$ may be mapped into a Gaussian state with $\mathrm{CM} \sigma_{f}=\nu_{f} S D_{\varphi_{f}} \operatorname{diag}\left(z_{f}, 1 / z_{f}\right) D_{\varphi_{f}}^{\top} S^{\top}$ via a GTO at inverse temperature $\beta$ if and only if

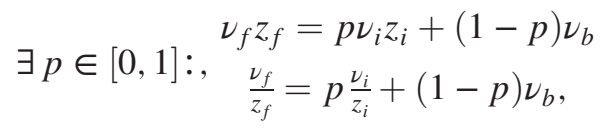

with $\nu_{b}=\left(\mathrm{e}^{\beta \omega}+1\right) /\left(\mathrm{e}^{\beta \omega}-1\right)$.

Note that the parameters $\varphi_{i}$ and $\varphi_{f}$ are irrelevant to the transformation criterion, which admits a simple geometrical representation: if one parametrizes the class of single-mode Gaussian states (in the basis of normal modes of $\hat{H}_{s}$ and modulo phase shifters) in the two-dimensional space $(\nu z, \nu / z)$, one can thermally map the states $\left(\nu_{i} z_{i}, \nu_{i} / z_{i}\right)$ only into states lying along the segment connecting $\left(\nu_{i} z_{i}, \nu_{i} / z_{i}\right)$ to $\left(\nu_{b}, \nu_{b}\right)$ (see [30]). Note that the squeezed states to which this criterion applies display quantum coherence (off-diagonal elements) in the energy eigenbasis. In the case with no squeezing, where the states have no coherence in the energy eigenbasis, the transformation criterion reduces to $\nu_{f} \in\left[\nu_{b}, \nu_{i}\right]$. In physical terms, this is equivalent to stating that GTOs send an initial thermal state at temperature $T_{i}$ into a final thermal state at temperature $T_{f}$ falling between $T_{i}$ and the environment's temperature $T$. This complies with the thermomajorization and the many second laws criteria of [36,37] (see [30]), while the case with squeezing falls beyond the criteria's scope. Interestingly, the prediction that $T_{f}$ must fall between $T_{i}$ and $T$ differs from what happens in qubit systems and turns out to be crucial for the task of cooling, to which we now turn.

Algorithmic cooling.- Let us now discuss the main repercussions of the characterization derived above on the algorithmic cooling of Gaussian systems. In the spirit of heat-bath algorithmic cooling (HBAC) [38] one aims at cooling a system by alternating Gaussian unitaries and thermal operations which, if one allows for partial rather than complete thermalizations, may lead to improvements in the cooling of finite-dimensional systems [27,39]. For example, a single qubit can be cooled arbitrarily close to the ground state by applying to it Pauli $x$ unitaries interspersed with thermal operations, without the need of extra ancillas. In fact, at low enough temperatures, the required thermal operations can be approximated by resonant Jaynes-Cummings couplings to a single, initially thermal oscillator. A natural question is then if a single system oscillator can be cooled below the environment temperature in a similar fashion, that is, by unitaries on the system acting between the GTOs. This would be particularly advantageous because it would only require standard quadratic interaction Hamiltonians.

Here we answer this question in the negative for singlemode systems: if $\mathcal{U}_{j}$ are single-mode (not necessarily Gaussian) unitaries and $\mathcal{T}_{j}$ arbitrary single-mode GTOs, for each $N$ the state $\mathcal{T}_{N} \circ \mathcal{U}_{N} \circ, \ldots, \mathcal{T}_{1} \circ \mathcal{U}_{1}\left[\varrho_{0}\right]$ cannot be cooled below the minimum between the environment's entropy and the initial system entropy. This is the case since the output entropy of phase-covariant, single-mode Gaussian channels at given input entropy is minimized by (Gaussian) thermal inputs (with respect to the normal mode Hamiltonian) [40], with optimal output entropy that is monotonic in the input entropy. Thus, the best the unitaries $\mathcal{U}_{j}$ can do is put the state in normal form which, for given initial symplectic eigenvalue $\nu_{j}$, yields the output symplectic eigenvalue $p \nu_{j}+(1-p) \nu_{b} \geq \min \left\{\nu_{j}, \nu_{b}\right\}$, so that the minimum entropy is obtained by either shielding completely from the environment or by complete thermalization. Notice that, rather remarkably, such an entropic bound holds for any unitary operation and any input state, not necessarily Gaussian. We also show in the Supplemental Material [30] that the impossibility of lowering the system entropy below the environment's value is maintained if one extends the class of thermal operations to include single-mode squeezed baths, which are not encountered spontaneously in nature but may be engineered under certain controlled conditions [41-45].

Cooling opportunities open up if nonquadratic interaction Hamiltonians or control over the energy levels' structure are allowed, as is commonly assumed for quantum refrigerators [46], or if some of the thermal ancillary modes can be manipulated by general Gaussian unitaries. In point of fact, these latter schemes, unless restricted by practical constraints, allow one to always cool any oscillator arbitrarily close to the ground state. To this aim, one may in principle include a thermal ancillary mode at high enough frequency so that its entropy is arbitrarily low, and then swap such a low entropy state into the system through a beam splitter acting in the unitary step (notice that such an interaction between modes at different frequencies would not be prohibited, since the unitary does not have to be a thermal operation in the general setup we are considering). This is nothing but the discrete version of sideband cooling, where excitations are extracted from the system of interest (such as a mechanical oscillator) into a coupled oscillator (such as a mode of light, in optomechanical setups) at higher frequency, from where they leak to the environment.

Our no-go theorem complements the impossibility of engineering absorption refrigerators with Gaussian resources alone, pointed out in [47]. Our treatment is more broader, relying on the general GTOs rather than on a 
specific time-evolution, and focuses on the system temperature, rather than heat transport between reservoirs.

Conclusions and outlook.-We presented a full characterization of Gaussian thermal operations, implying that they are all generated by a simple, time-local master equation, determined necessary and sufficient conditions for transformation under GTO on a single-mode and proved that no algorithmic cooling acting on a single-mode system alone can ever lower the entropy below the background or initial ones, a fact which is relevant in practice given the broad applicability of noise models based on bilinear interactions with an environment. The latter finding is intimately related to the fact that GTOs are all Markovian. As such, any dynamical trajectory reaching the thermal state must terminate there. In fact, the cooling protocol for a single qubit presented in [27] relied precisely on the fact that system-bath correlations can be used to cross the thermal state and achieve temperatures lower than that of the environment. This possibility is precluded, for Gaussian systems, by our no-go result. Our framework, however, sets up the scene to explore transformation conditions and more articulate cooling schemes in multimode scenarios (we refer the reader to the final section of the Supplemental Material [30] for a detailed discussion of future perspectives).

M. Genoni played a key role by liaising between the first two authors. Discussions with H. Jee, C. Sparaciari, G. De Palma and M. Huber, A. Levy and M. Mehboudi are also warmly acknowledged. M. L. acknowledges financial support from the European Union's Marie SkłodowskaCurie individual Fellowships (H2020-MSCA-IF-2017, GA794842), Spanish MINECO (Severo Ochoa SEV2015-0522 and Project No. QIBEQI FIS2016-80773-P) and Fundacio Cellex and Generalitat de Catalunya (CERCA Programme and SGR 875). G. A. acknowledges financial support from the European Research Council under the Starting Grant GQCOP (Grant No. 637352), as well as from FAPESP (Grant No. 2017/07973-5).

Note added.-During the completion of this Letter, we became aware of closely related work [48], where thermal transformations are constrained to passive unitaries by design and several multimode necessary conditions for state transformation are discussed.

[1] Thermodynamics in the Quantum Regime: Fundamental Aspects and New Directions, edited by F. Binder, L. A. Correa, C. Gogolin, J. Anders, and G. Adesso (Springer, Cham, 2019).

[2] J. Goold, M. Huber, A. Riera, L. del Rio, and P. Skrzypczyk, J. Phys. A 49, 143001 (2016).

[3] S. Vinjanampathy and J. Anders, Contemp. Phys. 57, 545 (2016).
[4] R. Alicki and R. Kosloff, in Thermodynamics in the Quantum Regime: Fundamental Aspects and New Directions (Springer, Cham, 2019), Chap. 1.

[5] D. Janzing, P. Wocjan, R. Zeier, R. Geiss, and T. Beth, Int. J. Theor. Phys. 39, 2717 (2000).

[6] F. G. S. L. Brandão, M. Horodecki, J. Oppenheim, J. M. Renes, and R. W. Spekkens, Phys. Rev. Lett. 111, 250404 (2013).

[7] M. Lostaglio, Rep. Prog. Phys. 82, 114001 (2019).

[8] N. Ng and M.P. Woods, in Thermodynamics in the Quantum Regime: Fundamental Aspects and New Directions (Springer, Cham, 2019), Chap. 26.

[9] M. Brunelli, A. Xuereb, A. Ferraro, G. De Chiara, N. Kiesel, and M. Paternostro, New J. Phys. 17, 035016 (2015).

[10] A. Mari, A. Farace, and V. Giovannetti, J. Phys. B 48, 175501 (2015).

[11] M. Brunelli, L. Fusco, R. Landig, W. Wieczorek, J. Hoelscher-Obermaier, G. Landi, F. L. Semião, A. Ferraro, N. Kiesel, T. Donner, G. De Chiara, and M. Paternostro, Phys. Rev. Lett. 121, 160604 (2018).

[12] G. Huber, F. Schmidt-Kaler, S. Deffner, and E. Lutz, Phys. Rev. Lett. 101, 070403 (2008).

[13] S. An, J.-N. Zhang, M. Um, D. Lv, Y. Lu, J. Zhang, Z.-Q. Yin, H. T. Quan, and K. Kim, Nat. Phys. 11, 193 (2015).

[14] E. G. Brown, N. Friis, and M. Huber, New J. Phys. 18, 113028 (2016).

[15] N. Friis and M. Huber, Quantum 2, 61 (2018).

[16] S. Deffner and E. Lutz, Phys. Rev. E 77, 021128 (2008)

[17] U. Singh, M. G. Jabbour, Z. Van Herstraeten, and N. J. Cerf, Phys. Rev. A 100, 042104 (2019).

[18] A. Belenchia, L. Mancino, G. T. Landi, and M. Paternostro, arXiv:1908.09382.

[19] A. Dhar, Adv. Phys. 57, 457 (2008).

[20] L. A. Correa, M. Perarnau-Llobet, K. V. Hovhannisyan, S. Hernández-Santana, M. Mehboudi, and A. Sanpera, Phys. Rev. A 96, 062103 (2017).

[21] M. Mehboudi, J. M. R. Parrondo, and A. Acín, New J. Phys. 21, 083036 (2019).

[22] L. Lami, B. Regula, X. Wang, R. Nichols, A. Winter, and G. Adesso, Phys. Rev. A 98, 022335 (2018).

[23] B. Yadin, F. C. Binder, J. Thompson, V. Narasimhachar, M. Gu, and M. S. Kim, Phys. Rev. X 8, 041038 (2018).

[24] D. Walls and G. J. Milburn, Quantum Optics (SpringerVerlag, Berlin Heidelberg, 2008).

[25] A. Serafini, Quantum Continuous Variables: A Primer of Theoretical Methods (CRC Press, Boca Raton, FL, USA, 2017).

[26] K. Sala and T. Tufarelli, Sci. Rep. 8, 9157 (2018).

[27] Á. M. Alhambra, M. Lostaglio, and C. Perry, Quantum 3, 188 (2019).

[28] G. Adesso, S. Ragy, and A. R. Lee, Open Syst. Inf. Dyn. 21, 1440001 (2014).

[29] V. Giovannetti, R. Garcia-Patron, N. J. Cerf, and A. S. Holevo, Nat. Photonics 8, 796 (2014).

[30] See Supplemental Material at http://link.aps.org/ supplemental/10.1103/PhysRevLett.124.010602 for a detailed treatment, which includes Refs. [31-34].

[31] H. Führa and Z. Rzeszotnik, Linear Algebra Appl. 547, 32 (2018). 
[32] A. Mari, V. Giovannetti, and A. S. Holevo, Nat. Commun. 5, 3826 (2014).

[33] V. Giovannetti, A. S. Holevo, and R. García-Patrón, Commun. Math. Phys. 334, 1553 (2015).

[34] M. P. Muller, Phys. Rev. X 8, 041051 (2018).

[35] Whilst the environment may be set in normal form wlog, since thermal maps do not depend on the choice of environmental basis.

[36] M. Horodecki and J. Oppenheim, Nat. Commun. 4, 2059 (2013).

[37] F. G. S. L. Brandão, M. Horodecki， N. H. Y. Ng， J. Oppenheim, and S. Wehner, Proc. Natl. Acad. Sci. U.S.A. 112, 3275 (2015).

[38] D. K. Park, N. A. Rodriguez-Briones, G. Feng, R. Rahimi, J. Baugh, and R. Laflamme, in Electron Spin Resonance (ESR) Based Quantum Computing (Springer, New York, 2016), pp. 227-255.

[39] N. A. Rodriguez-Briones, J. Li, X. Peng, T. Mor, Y. Weinstein, and R. Laflamme, New J. Phys. 19, 113047 (2017).
[40] G. De Palma, D. Trevisan, and V. Giovannetti, Phys. Rev. Lett. 118, 160503 (2017).

[41] P. Tombesi and D. Vitali, Phys. Rev. A 50, 4253 (1994).

[42] N. Lütkenhaus, J. I. Cirac, and P. Zoller, Phys. Rev. A 57, 548 (1998).

[43] T. Werlang, R. Guzmán, F. O. Prado, and C. J. Villas-Bôas, Phys. Rev. A 78, 033820 (2008).

[44] A. Kronwald, F. Marquardt, and A. A. Clerk, New J. Phys. 16, 063058 (2014).

[45] J. Klaers, S. Faelt, A. Imamoglu, and E. Togan, Phys. Rev. X 7, 031044 (2017).

[46] R. Kosloff and A. Levy, Annu. Rev. Phys. Chem. 65, 365 (2014).

[47] E. A. Martinez and J. P. Paz, Phys. Rev. Lett. 110, 130406 (2013).

[48] V. Narasimhachar, S. Assad, F. C. Binder, J. Thompson, B. Yadin, and M. Gu, arXiv:1909.07364. 